\title{
The CRZ1/SP1-like gene links survival under limited aeration, cell integrity and biofilm formation in the pathogenic yeast Cryptococcus neoformans
}

\author{
Zuzana Moranovaa,b, Eric Virtudazo ${ }^{b}$, Kristyna Hricova ${ }^{a}$, Misako Ohkusu ${ }^{b}$, Susumu Kawamoto ${ }^{b}$, Vendula Husickovaa, \\ Vladislav Raclavsky ${ }^{\mathrm{a}}$
}

\begin{abstract}
Aims. Limited aeration has been demonstrated to cause slowdown in proliferation and delayed budding, resulting eventually in a unique unbudded G2-arrest in the obligate aerobic pathogenic yeast Cryptococcus neoformans. Also, the ability to adapt to decreased oxygen levels during pathogenesis has been identified as a virulence factor in C. neoformans. The aim of this study was to identify and characterize genes that are necessary for the proliferation slowdown and G2-arrest caused by limited aeration.

Methods. Random mutants were prepared and screened for lack of typical slowdown of proliferation under limited aeration. The CNAG_00156.2 gene coding for a zinc-finger transcription factor was identified in mutants showing most distinctive phenotype. Targeted deletion strain and reconstituted strain were prepared to characterize and confirm the gene functions. This gene was also identified in a parallel studies as homologous both to calcineurin responsive (Crz1) and PKC1-dependent (SP1-like) transcription factors.

Results. We have confirmed the role of the cryptococcal homologue of CRZ1/SP1-like transcription factor in cell integrity, and newly demonstrated its role in slowdown of proliferation and survival under reduced aeration, in biofilm formation and in susceptibility to fluconazole.

Conclusions. Our data demonstrate a tight molecular link between slowdown of proliferation during hypoxic adaptation and maintenance of cell integrity in C. neoformans and present a new role for the CRZ1 family of transcription factors in fungi. The exact positioning of this protein in cryptococcal signalling cascades remains to be clarified.
\end{abstract}

Key words: Cryptococcus, hypoxia, G2 arrest, CRZ1, SP1, cell integrity, capsule, biofilm, fluconazole, dormant infection

Received: November 26, 2012; Accepted with revision: March 25, 2013; Available online: April 22, 2013 http://dx.doi.org/10.5507/bp.2013.024

${ }^{a}$ Department of Microbiology, Faculty of Medicine and Dentistry, Palacky University Olomouc, Czech Republic ${ }^{b}$ Division of Molecular Biology, Medical Mycology Research Center, Chiba University, Chiba, Japan Corresponding author: Vladislav Raclavsky, e-mail: vladislav.raclavsky@upol.cz

\section{INTRODUCTION}

For Cryptococcus neoformans to cause disease, it must adapt to the host environment, which is very different from its natural habitat. C. neoformans has three well established virulence factors: a capsule, melanin and the ability to grow at human body temperature ${ }^{1}$. Recently, the ability to adapt to decreased oxygen levels during pathogenesis has emerged as another virulence factor in this obligate aerobic yeast species. It is generally accepted that well ventilated lungs are the primary site of cryptococcal infection, typically followed by spread to central nervous system (CNS). Other organs are affected by cryptococcal infection rather rarely. Oxygen was demonstrated to be a growth-limiting nutrient for $C$. neoformans ${ }^{2}$ and deficit in oxygen under limited aeration was demonstrated to delay cryptococcal budding resulting eventually in a unique unbudded G2-arrest ${ }^{3-5}$.

When searching for genes important for hypoxic adaptation, Chun et al. isolated hypoxia sensitive mutants harbouring mutations in three homologs of proteins that act in the mammalian SREBP pathway, namely cryptococ- cal SREBP homologue Sre1, Site-2-protease homologue Stp1, SCAP homologue Scp1, and also Tco1, a member of a highly conserved family of fungal-specific hybrid histidine kinases ${ }^{6}$. These mutants exhibit defect in growth under hypoxic conditions, but not under normoxic conditions. In parallel with the work of Chun et al., Chang et al. confirmed these observations in sre 1 and scp 1 deletion mutants ${ }^{7}$ and described several new members of this pathway $^{8}$. Mutants in either the SREBP pathway or the Tco 1 pathway display defects in their ability to proliferate in host tissues and to cause disease in infected mice, linking hypoxic adaptation and pathogenesis. For recent reviews on this topics see also ref. ${ }^{9-12}$.

Here we report that the cryptococcal CRZ1/SPI-like gene plays an important role not only in cell wall integrity, but also in delay of cell cycle progression, survival under limited aeration, and in biofilm formation in the pathogenic yeast $C$. neoformans. This represents novel biological roles for this conserved protein and also opens a new field in the study of hypoxia sensing and signalling in C. neoformans. 


\section{MATERIALS AND METHODS}

\section{Strains and cultivation}

Cryptococcus neoformans var. grubii (serotype A) strain IFM49144 showing typical unbudded G2-arrest ${ }^{4}$ was from Culture Collection of the Medical Mycology Research Center, Chiba University, Chiba, Japan. In-house prepared YPG medium (10 g/L of each yeast extract, peptone, and glucose) was used for liquid cultivation, YPG plates with appropriate supplements were used for phenotypical analysis. Rice extract agar and Sabouraud agar were from Trios (Prague, Czech Republic) and Caffeic Acid Ferric Citrate (CAFC) Agar was from Himedia Laboratories (Mumbai, India). For controlled hypoxic conditions plates were incubated in a $1 \% \mathrm{O}_{2}, 10 \% \mathrm{CO}_{2}$, 9.4\% $\mathrm{H}_{2}$, and $79.3 \% \mathrm{~N}_{2}$ atmosphere using an Anoxomat system (Mart Microbiology, Drachten, Netherlands).

Agrobacterium mediated random insertional mutagenesis

Random insertional mutagenesis of $C$. neoformans was performed by transformation with $A$. tumefaciens harbouring kanamycin resistance plasmid pYCC716. The cocultivation experiments were performed as described earlier ${ }^{13}$. Briefly, C. neoformans cells were co-cultivated with $A$. tumefaciens for 3 days at $24{ }^{\circ} \mathrm{C}$ on co-cultivation medium $(\mathrm{CCM})$ at the $1 \mathrm{C}: 10 \mathrm{~A}$ ratio. The bacteria/yeast mixture was scratched from CCM and resuspended in sterile water. Aliquots were plated on YPG agar containing 200 $\mu \mathrm{g} / \mathrm{mL}$ G418 (Sigma Aldrich) and $200 \mathrm{mM}$ cefotaxime (Alchemica). After 2-day cultivation 665 transformants were obtained.

\section{Screening for mutants showing hypoxia-specific phenotypes}

Transformants were screened according to hypoxia response with regard to cell cycle progression to identify components of hypoxia-induced G2 arrest on molecular level. Previously optimized high-throughput arrangement of experiments was used to induce typical unbudded G2arrest under moderate hypoxia compared to unrestricted normoxic cell cycle progression ${ }^{14}$. Briefly, cultivation was performed in 24-well cell culture plates sealed with SecureSeal adhesive film (Simport) which is less than $0.01 \%$ oxygen permeable and filled with $300 \mu \mathrm{L}$ of YPG media to secure good aeration for normoxic conditions and $600 \mu \mathrm{L}$ for reduced aeration resulting in moderately hypoxic conditions. The plates were shaken reciprocally at $200 \mathrm{rpm}$ at $30{ }^{\circ} \mathrm{C}$ for $48 \mathrm{~h}$ and cell count was measured using a Multisizer 3 Coulter Counter (Beckman Coulter, Brea, California, USA). To select those insertional mutants that lacked typical response, degree of proliferation slowdown in response to reduced aeration was calculated as the ratio of the number of cells grown at moderately reduced aeration $(600 \mu \mathrm{L})$ to the number of cells grown at good aeration $(300 \mu \mathrm{L})$. Thus, higher ratio indicated lower degree of slowdown in response to reduced aeration. The insertional mutant showing highest ratio was selected for identification of the insertional locus.

\section{Identification of the insertional locus}

Thermal asymmetric interlaced-PCR (TAIL-PCR) (ref. ${ }^{15}$ ) was performed to gain genomic sequences adjacent to the inserted DNA. Amplicons were sorted in 2\% (w/v) agarose gel, purified using QIAquick gel extraction Kit (Qiagen) and sequenced using a BigDye Terminator v3.1 Cycle Sequencing Ready Reaction Kit (Applied Biosystems) with an ABI Prism 3130xl Genetic Analyzer apparatus (Applied Biosystems) in accordance with manufacturer's instructions. Acquired sequences were searched for homology using the BLAST algorithm in C. neoformans var. grubii H99 genomic sequence (www. broadinstitute.org) to identify the insertional locus.

\section{Targeted deletion strategy}

Overlap extension PCR was used to create overlap fragment for biolistic transformation as already described ${ }^{16}$. The primer pairs TF1.1F/TF2R and TF3F/TF4.1R were designed and used with genomic DNA to obtain the left (1 kb upstream of the CNAG_00156.2 gene) and right ( $1 \mathrm{~kb}$ downstream of the same gene) arms for the disruption construct. The selectable marker NEO was amplified using the primers M13F and M13R from the plasmid

Table 1. List of primers used in the study.

\begin{tabular}{ll}
\hline Name & Sequence \\
\hline KT8.1F & GGGTTTAAGACGAAAGAAAG \\
KT8.1R & GATTGCCTTTCCCGACTTTG \\
KT8.2R & CAGCAGGATTGTAATCTGTG \\
M13F & GTAAAACGACGGCCAG \\
M13R & CAGGAAACAGCTATGAC \\
NAT-F & TGGATCAACAGGGACTTCTC \\
NAT-R & ACTCGCCCAACATGTCTATG \\
TF1.1F & CAACTGCCGCATCATTTGCA \\
TF1.3F & GAGCAGATTGTGTATCTTGC \\
TF1.3F LP & CGTGAGTAACTTTTCCTTCG \\
TF2R & GTCATAGCTGTTTCCTGTTTTTGTGGATTATAGGGGTGA \\
TF3F & CTGGCCGTCGTTTTACTCGCCCGATGGTCATAGGGCGCTG \\
TF3R+M13R & gtcatagctgtttcctgTTAATCCTCTTCACTCGTTT \\
TF4.1R & GAAGTCGAGGCGCAGTGATT \\
\hline
\end{tabular}


pJAF1, which contains the neomycin resistance marker cassette for $C$. neoformans. Primers TF1.1F and TF4.1R were used to create an overlap fragment $(1 \mu \mathrm{L}$ of each amplified fragment); see Table 1 for list of primers. All PCR reactions were performed with KOD Plus (Toyobo) DNA polymerase $(0.1 \mathrm{U} / \mu \mathrm{L})$ using $0.5 \mu \mathrm{M}$ primers, 0.2 $\mathrm{mM}$ dNTPS, $1.5 \mathrm{mM} \mathrm{Mg}^{2+}$ and amplified using an $\mathrm{iCy}-$ cler Thermal Cycler (BioRad) with initial denaturation at $94^{\circ} \mathrm{C}$ for $4 \mathrm{~min}$, followed by 30 cycles of denaturation at $94{ }^{\circ} \mathrm{C}$ for $30 \mathrm{~s}$, annealing at $55^{\circ} \mathrm{C}$ for $30 \mathrm{~s}$, and extension at $68{ }^{\circ} \mathrm{C}$ for $6 \mathrm{~min}$, with final extension at $68^{\circ} \mathrm{C}$ for 10 min. To confirm overlap reaction, the PCR product was run on $1 \%(\mathrm{w} / \mathrm{v})$ agarose gel and overlap fragment was cut out of the gel and purified using QIAquick gel extraction Kit (Qiagen). The resulting 4-kb overlap PCR product was used to transform the IFM49144 strain by biolistic transformation as described below. Three independent transformants were screened for proper targeted deletion by colony PCR using primers KT8.1F and KT8.1R which were designed outside of overlap sequence resulting in a $5978 \mathrm{bp}$ fragment in control strain harbouring the CNAG_00156.2 (CRZ1/SPl-like) gene and in a 4367 bp fragment in the targeted deletion strain with neomycin resistance gene. Proper deletion was also confirmed by Southern blot analysis described below.

\section{Reconstitution of CNAG_00156.2 (CRZ1/SP1-like) gene targeted deletion strain}

Primers TF1.3F and TF3R+M13R were used to amplify $1 \mathrm{~kb}$ region upstream of the CNAG_00156.2 (CRZ1) SPl-like) gene stop codon, and primers $\mathrm{TF} 3 \mathrm{~F}+\mathrm{M} 13 \mathrm{~F}$ and TF4.1R to amplify $1 \mathrm{~kb}$ region downstream of the same stop codon from IFM49144 strain genomic DNA. These two fragments were PCR overlapped with the NAT marker (M13R-M13F direction) and the overlap product (4042 bp) was used to transform the IFM49144 wild type strain. Primers TF1.3F LP and KT8.1R were used to screen for correct transformants that have the NAT marker inserted downstream of the CNAG_00156.2 gene (4184 bp in NAT transformants compared to $2269 \mathrm{bp}$ in wild type). The genomic copy of the CNAG_00156.2 (CRZ1/SPl-like) gene amplified by TF1.1F and KT8.1R primers was fused with the NAT marker (7807 bp). Purified PCR product was used to transform the CNAG_00156.2del::NEO targeted deletion strain. Primers KT8.1F and KT8.2R were used to screen for three independent correct transformants (7979 bp in CNAG_00156.2-NAT reconstituted transformants compared to 4466 bp in the CNAG_00156.2del::NEO targeted deletion strain and $6064 \mathrm{bp}$ in the wild type strain) and also paired KT8.1F with NAT-R and KT8.2R with NAT-F to have an internal PCR check with NAT marker. See Table 1 for list of primers.

\section{Biolistic transformations}

The procedure described earlier ${ }^{17}$ was modified and optimized empirically to achieve best transformation rate in the IFM 49144 strain. Briefly, $1 \mathrm{~mL}$ of an overnight culture in YPG was pelleted and resuspended in $0.5 \mathrm{~mL}$ of fresh YPG. Approximately $200 \mu \mathrm{L}$ of the cell suspension were spread per each minimal medium plate with
$1 \mathrm{M}$ sorbitol and plates were air-dried. DNA samples were prepared by combining $600 \mu \mathrm{g}$ of gold microcarrier beads $(0.6 \mu \mathrm{m}$ in diameter, Bio-Rad) with $1-4 \mu \mathrm{g}$ of the overlap DNA fragment, $10 \mu \mathrm{L}$ of $2.5 \mathrm{M} \mathrm{CaCl}_{2}$, and $2 \mu \mathrm{L}$ of $1 \mathrm{M}$ spermidine. This mixture was incubated for 2-3 min at room temperature while vortexing vigorously, followed by $10 \mathrm{~min}$ incubation without agitation. Beads were centrifuged, washed with $30 \mu \mathrm{L}$ of $70 \%$ ethanol followed by $30 \mu \mathrm{L}$ of $100 \%$ ethanol, and then resuspended in $12 \mu \mathrm{L}$ of $100 \%$ ethanol. The solution was placed on macrocarrier disks (Bio-Rad) and YPG plates supplemented with $1 \mathrm{M}$ sorbitol (without antibiotics) were then bombarded with the DNA-bound gold microcarrier beads in a PDS1000 biolistic transformation apparatus (Bio-Rad) at a helium gas pressure of approximately 1350 psi under a vacuum of 29 in of $\mathrm{Hg}$. After the transformation plates were incubated for $4 \mathrm{~h}$ at $30^{\circ} \mathrm{C}$, the cells were harvested and resuspended in $200 \mu \mathrm{L}$ of PBS and spread on plates supplemented with $200 \mu \mathrm{g} / \mathrm{mL}$ of G418, followed by further cultivation at $30{ }^{\circ} \mathrm{C}$ for 3-5 days.

\section{Southern Blot Analysis}

Southern blot analysis for verification of single targeted deletion and reconstitution was performed as follows: C. neoformans genomic DNA $(\sim 10 \mu \mathrm{g})$ was digested with restriction enzyme BgIII, fractionated by electrophoresis in $1 \%$ agarose gel, transferred to a nylon membrane, and fixed by UV crosslinking. The membrane was hybridized overnight with $1 \mathrm{~kb}$ DNA fragment prepared with PCR DIG Probe Synthesis Kit as probe and the membranes were washed, dried and exposed to film.

\section{Cell cycle and cell death assays}

Cells were fixed by ethanol and stained by PicoGreen fluorescent dye (Invitrogen) diluted 1:500. PicoGreen shows high affinity to dsDNA, making the RNase treatment unnecessary compared to conventional PI staining, and it also surpasses performance of the dsDNA selective dye Hoechst 33258 ( ref. $^{18}$ ). This is particularly useful in $C$. neoformans, where the cell cycle analysis based on DNA content measurement can be tedious because of the less permeable cell wall and variable background fluorescence. PicoGreen has already been used for cell cycle assays in bacteria; however, this is its first use for such purpose in yeasts to our knowledge. After washing with PBS, proper staining of nuclei in contrast to low cytoplasmic background was checked under fluorescence microscope. If needed, dilution of PicoGreen dye was adjusted to reduce background or increase fluorescence of nuclei for proper DNA content monitoring. A Cytomics FC 500 flow cytometer (Beckmann-Coulter) was used for measurement and cell cycle analysis was performed manually in plots of PicoGreen fluorescence vs. forward scatter based on cell size and fluorescence intensity. A cell death rate assay already described in C. albicans ${ }^{19}$ was modified as follows: cells were suspended in $0.01 \%$ Eosin $\mathrm{Y}$ in $1 \mathrm{M}$ sorbitol, observed under fluorescence microscope, photographed and percentage of dead cells was counted in at least 50 cells per sample. 


\section{Biofilm formation}

C. neoformans was grown on Sabouraud glucose agar (SGA) plates for $48 \mathrm{~h}$, a suspension in Sabouraud broth was prepared ( $\sim 10^{7}$ cells $\left./ \mathrm{mL}\right), 200 \mu \mathrm{L}$ were aliquoted into wells of a microtiter plate and incubated at $30{ }^{\circ} \mathrm{C}$ for $48 \mathrm{~h}$. Nonadherent cells were then washed gently by tap water and adherent cells were fixed by $200 \mu \mathrm{L}$ of $99 \%$ methanol per well for $15 \mathrm{~min}$. After removing the fixative and air drying, adherent cells were stained by 160 $\mu \mathrm{L}$ of aqueous crystal violet solution per well for $10 \mathrm{~min}$. After gentle washing by tap water, $160 \mu \mathrm{L}$ of $33 \%$ acetic acid was added, shaken and colorimetric reading of the destaining solution was performed at $570 \mathrm{~nm}$. Four wells were processed in parallel in each strain and in negative control.

\section{Antifungal susceptibility testing}

Minimum inhibitory concentration of fluconazole was determined using E-test strips (bioMérieux, Marcy l'Etoile, France) on the Antimycotic Sensitivity Test Agar (Trios, Prague, Czech Republic) in accordance with manufacturer's instructions.

\section{RESULTS}

The $C R Z 1 / S P 1$-like gene was disrupted in the random mutant that lacked slowdown of cell cycle progression under reduced aeration

Altogether, 665 C. neoformans random mutants were prepared by Agrobacterium mediated random insertional mutagenesis. Slowdown of proliferation in response to reduced aeration is the hallmark of cryptococcal response to moderate hypoxia that results in a typical unbudded G2-arrest eventually. Altogether, 18 random insertional mutants were selected for further analysis. Sequencing revealed insertion in 12 unique locuses, one of the insertion mutants contained 2 inserts in 2 different locuses. We selected the random insertional mutant showing lowest degree of proliferation slowdown in response to moderately reduced aeration for further study. The targeted locus was present in two other random mutants and was identified as the CNAG_00156.2 gene based on homology search in C. neoformans var. grubii $\mathrm{H} 99$ genomic DNA database using BLAST (www.broadinstitute.org). When a translated protein sequence of this gene was used to search for homology in $S$. cerevisiae, the CRZ1 gene coding for a zinc-finger transcription factor was identified as a potential homologue. Reversed search for a $C R Z 1$ homologue in cryptococcal genome revealed the same gene, as already published independently when identification of a cryptococcal homologue of $A C E 2$ transcription factor was sought ${ }^{20}$. However, a report refuting CNAG_00156.2 to be a functional homologue of $C R Z 1$ and collecting evidence for its homology to the Sp family of transcription factors was published during finishing our study ${ }^{21}$. Another study that was published during revision of our manuscript demonstrated its product to be a bona fide target of calcineurin under non-starvation conditions and to be the true ortholog of fungal Crz1 proteins ${ }^{22}$. Therefore, we will further refer to the CNAG_00156.2 gene as to cryptococcal $C R Z 1 / S P 1$-like gene.

Cn CRZ1/SP1-like gene is required for survival under limited aeration and for slowdown of proliferation

The CRZ1/SPl-like gene was removed in our IFM49144 strain by targeted deletion strategy and a reconstituted strain was also prepared as described in Material and methods. Two different volumes of culture medium ( 500 and $1000 \mu \mathrm{L}$ ) were used to expose the culture of the targeted deletion strain to different degrees of aeration (moderately and severely reduced) in contrast to wellaerated normoxic control $(100 \mu \mathrm{L})$, all in shaken liquid cultures in 24-well cell culture plates as described earlier ${ }^{14}$. Death rate was evaluated using Eosin Y staining and fluorescence microscopy. A convincing detrimental effect of the targeted $C R Z 1 / S P 1$-like gene deletion on survival was observed already after $24 \mathrm{~h}$, with $18 \%$ of dead cells under moderately limited aeration and $58 \%$ of dead cells under severely limited aeration $(2 \times$ or $7 \times$ more compared to $8 \%$ of dead cells in well aerated control, respectively). This effect was almost completely reversed in reconstituted strain (Fig. 1). To verify the role of hypoxia in this effect, serial dilutions of cells were also incubated on YPG plates under 1\% oxygen atmosphere in Anoxomat, resulting in reduced growth of the targeted deletion strain compared to both wild type and reconstitution strain after 2 weeks of incubation (Fig. 2). Furthermore, a decrease in G2 fraction in response to moderately limited aeration was observed as a result of the targeted deletion, in contrast to an increase in $\mathrm{G} 2$ fraction at the same conditions observed in wild type, indicating an essential role of intact

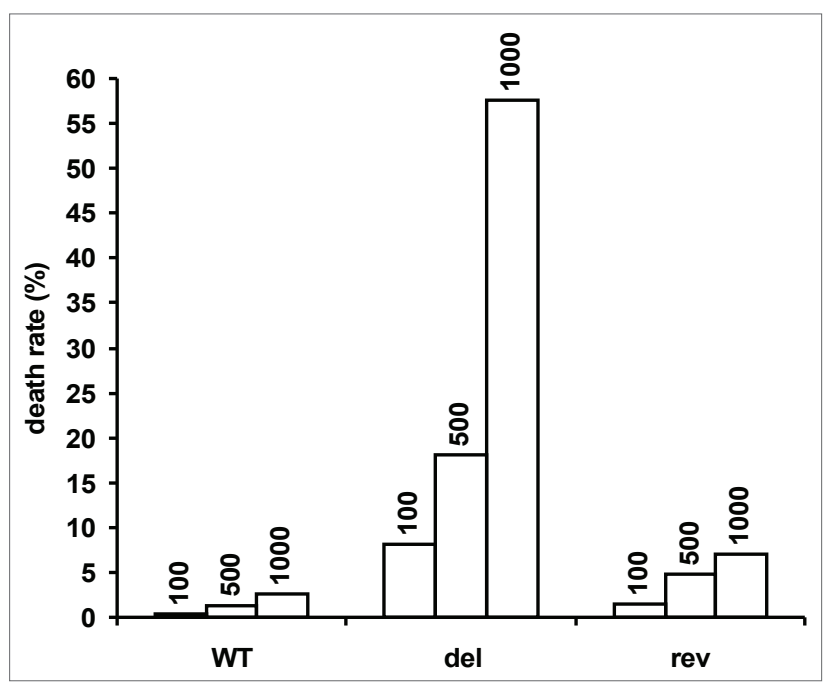

Fig. 1. Death rate under variable aeration.

Death rate after $24 \mathrm{~h}$ of cultivation in liquid shaken culture at $30^{\circ} \mathrm{C}$ under variable aeration - well aerated culture in $100 \mu \mathrm{L}$ volume (normoxic conditions), moderately limited aeration in $500 \mu \mathrm{L}$ (mild hypoxic conditions) and culture severely limited in aeration in $1000 \mu \mathrm{L}$ (profound hypoxic conditions); WT wild-type strain, del = CNAG_00156.2 (CRZ1/SP1-like) gene targeted deletion strain, rev $=$ reconstituted strain. 


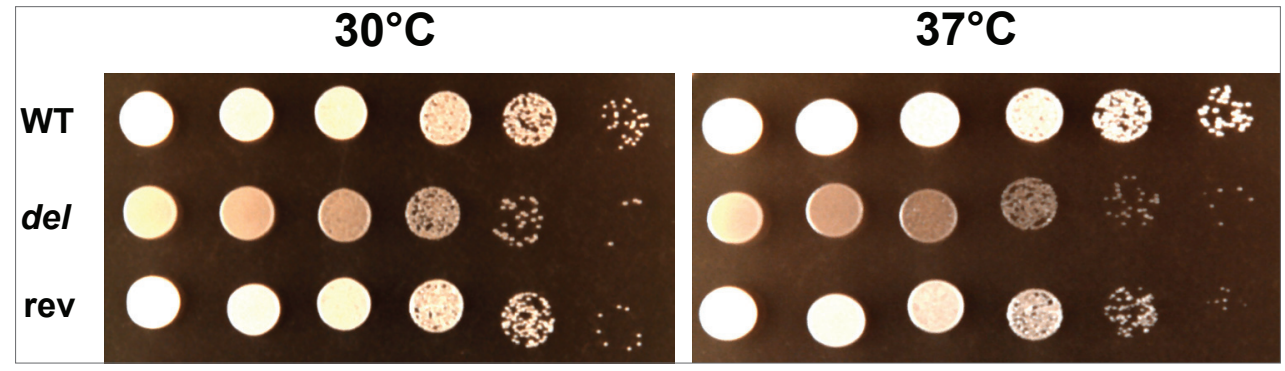

Fig. 2. Viability under reduced oxygen tension.

Aliquots of 10 -fold serial dilutions were plated onto YPG plates supplemented as indicated and cultured at 30 or $37{ }^{\circ} \mathrm{C}$ in a $1 \% \mathrm{O}_{2}, 10 \% \mathrm{CO}_{2}, 9.4 \% \mathrm{H}_{2}$, and $79.3 \% \mathrm{~N}_{2}$ atmosphere for 2 weeks. WT - wild-type strain, del = CNAG_00156.2 (CRZ1/SPl-like) gene targeted deletion strain, rev = reconstituted strain.

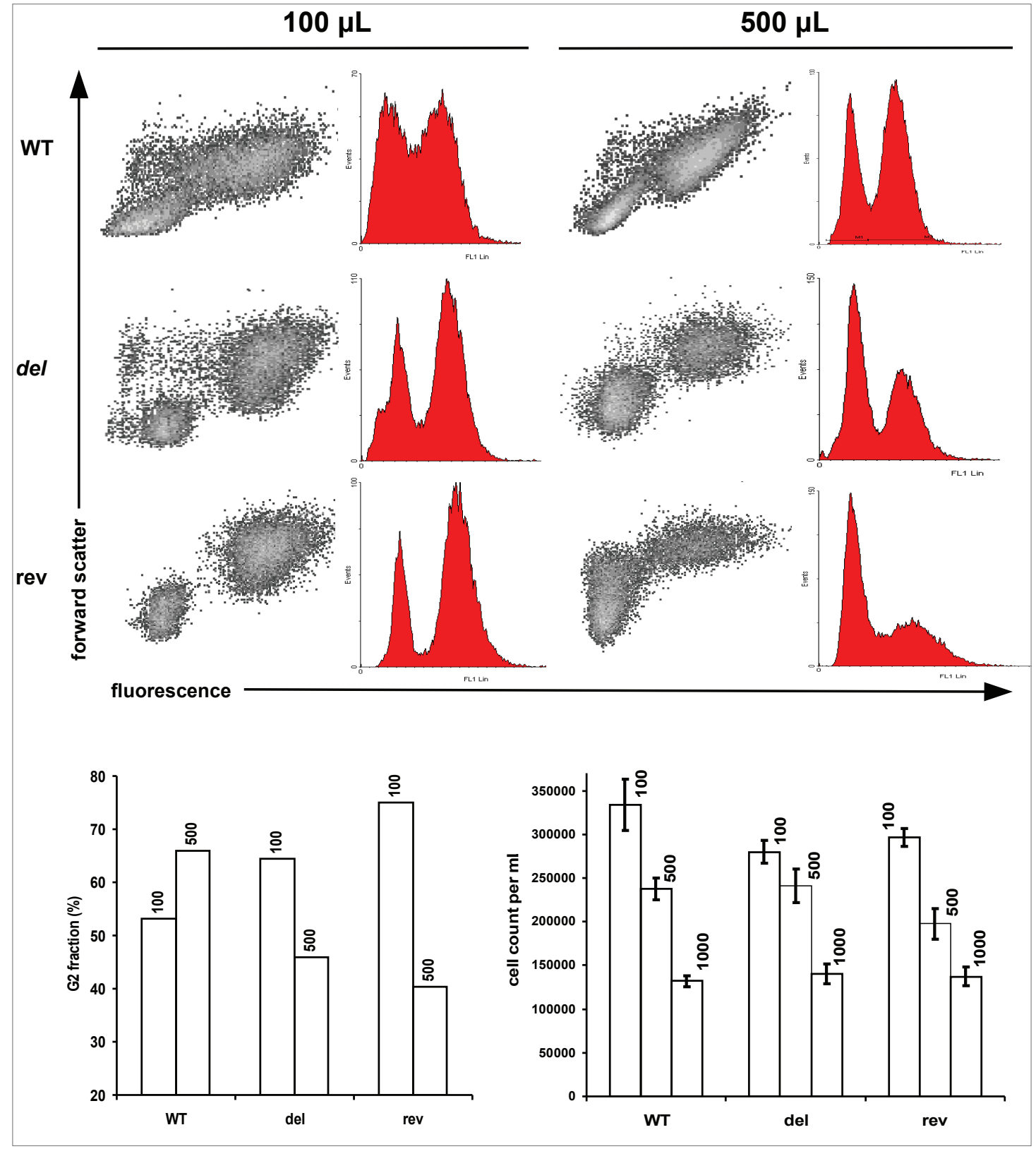

Fig. 3. Cell cycle analysis based on proliferation and DNA content assays.

Number of cells was monitored using Multisizer 3 Coulter Counter and DNA content was monitored in PicoGreen stained cells using a Cytomics FC 500 flow cytometer after $24 \mathrm{~h}$ of growth in well aerated $(100 \mu \mathrm{L})$ and moderately aerated $(500 \mu \mathrm{L})$ shaken liquid culture at $30^{\circ} \mathrm{C}$. WT - wild-type, del =CNAG_00156.2 (CRZ1) $S P$ l-like) gene targeted deletion strain, rev = reconstituted strain. 


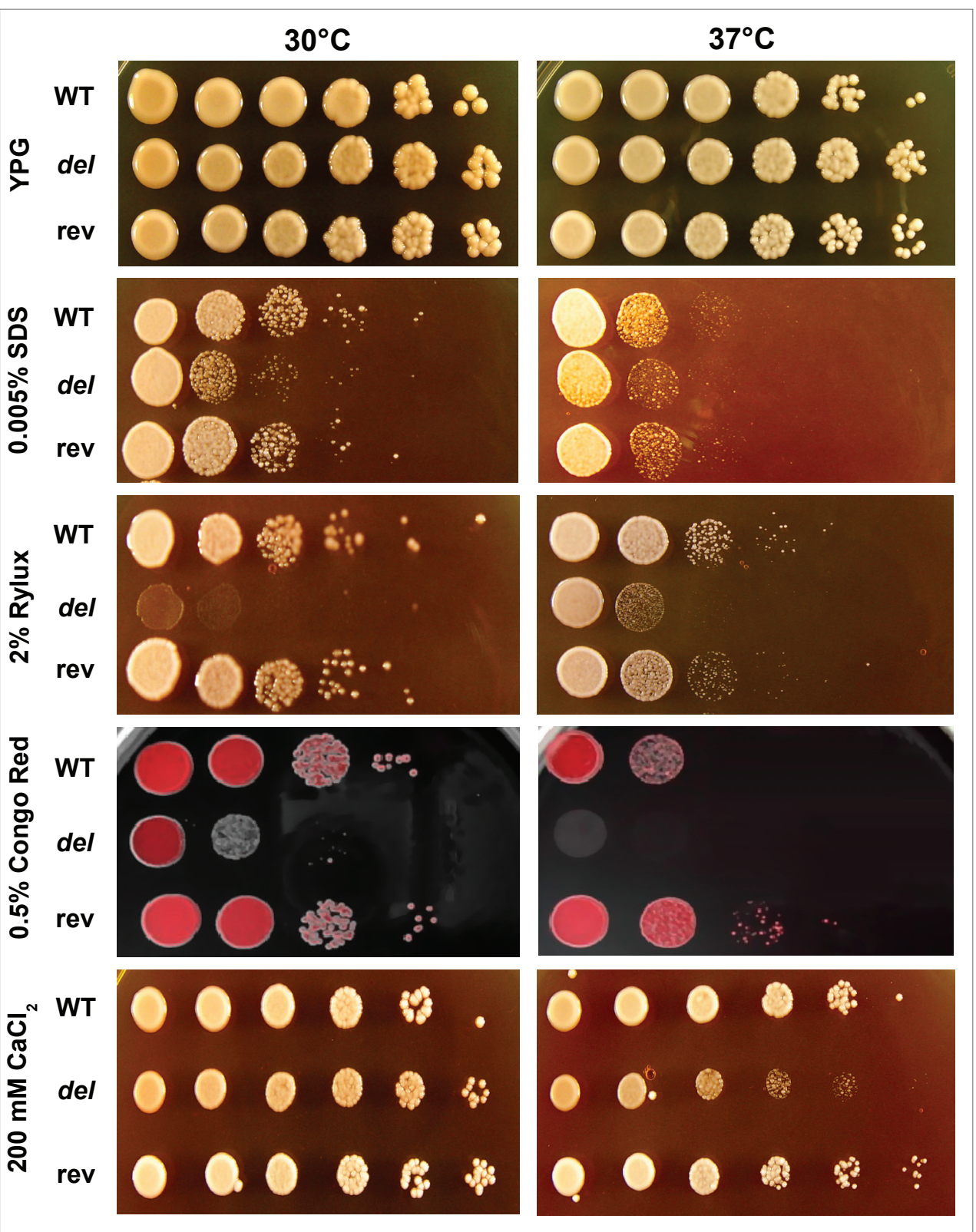

Fig. 4. Effect of cell integrity disturbing agents and calcium ions.

Aliquots of 10 -fold serial dilutions were plated onto YPG plates supplemented as indicated and cultured at 30 or $37^{\circ} \mathrm{C}$ for 3 , 6, or 8 days, depending on the particular plate. All spot assays were repeated at least three times, and representative experiments are shown. WT - wild-type, del $=$ CNAG_00156.2 (CRZ1/SP1-like) gene targeted deletion strain, rev = reconstituted strain.

$C R Z 1 / S P 1$-like gene in the hypoxia-induced $\mathrm{G} 2$ arrest in C. neoformans (Fig. 3). Accordingly, the cell number was reduced significantly under moderately reduced aeration compared to normoxic conditions in the wild type strain and reconstituted strain $(P=0.05)$, but not in the targeted deletion strain (Fig. 3). Because of the high death rate, conclusive interpretation of the effect of targeted CRZ1/ $S P 1$-like gene deletion on proliferation under severely reduced aeration was not possible. It was also impossible to evaluate the DNA content under severely reduced aeration, because of inconsistent staining and dead cells background. Further, reversion of the decreased G2 content under moderately reduced aeration was lacking in the reconstituted strain, most likely because the delicate cell cycle machinery was still somehow disturbed after reconstitution.

Cn $C R Z 1 / S P 1$-like gene is required for cell wall damage repair and for biofilm formation

Whereas no differences in growth were observed at elevated temperature $\left(37^{\circ} \mathrm{C}\right)$ on YPG plates, increased susceptibility of the targeted CRZ1/SPI-like gene deletion strain to cell wall damaging agents Congo Red, Rylux BSU (a Calcofluor White related compound, see ref. ${ }^{23}$ for details), and SDS were observed, both at 30 and $37^{\circ} \mathrm{C}$, as already described ${ }^{21}$ (Fig. 4). Increased susceptibility of the targeted deletion strain to calcium ions, but not to $\mathrm{LiCl}(150 \mathrm{mM})$ or $\mathrm{NaCl}(1.5 \mathrm{M})$, was not apparent 


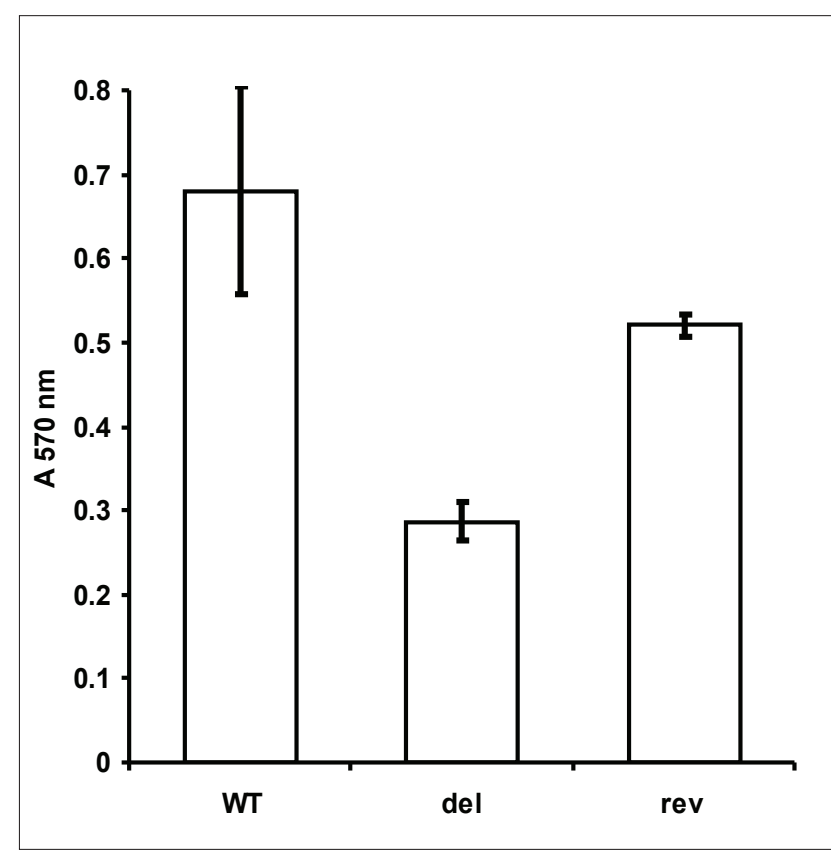

Fig. 5. Biofilm assay in polystyrene microtiter plates.

Cells were incubated in polystyrene microtiter plates at $30{ }^{\circ} \mathrm{C}$ for 2 days. Then, biofilm formation was detected by crystal violet staining after washing out nonadherent cells. Stained biofilm was quantified by measuring absorbance at $570 \mathrm{~nm}$. WT - wild-type, del = CNAG_00156.2 (CRZ1/SP1-like $)$ gene targeted deletion strain, rev $=$ reconstituted strain. at $30{ }^{\circ} \mathrm{C}$, but was unveiled at $37^{\circ} \mathrm{C}$ (Fig. 4). In addition to the role in cell wall damage repair, we also observed significantly reduced ability of the targeted deletion strain to form biofilm compared to wild type and reconstituted strain $(P=0.05$, Fig. 5$)$.

Cn CRZ1/SP1-like gene deletion results in stronger capsule induction and increased resistance to fluconazole

Whereas no apparent difference in melanin formation was observed on CAFC medium, significantly stronger induction of capsule formation was observed in the targeted $C R Z 1 / S P 1$-like gene deletion strain compared to wild type and reconstituted strain $(P=0.01)$ when grown on Rice extract agar under $5 \% \mathrm{CO}_{2}$ at $37{ }^{\circ} \mathrm{C}$ for 3 days. At the same time, no significant differences in cell diameter were observed (Fig. 6). Surprisingly, the targeted deletion strain also showed increased resistance to fluconazole (6 $\mu \mathrm{g} / \mathrm{mL})$ compared to wild type $(1.5 \mu \mathrm{g} / \mathrm{mL})$ and reconstituted strain $(3 \mu \mathrm{g} / \mathrm{mL})$ when minimum inhibitory concentration of fluconazole was determined using E-test strips.

\section{DISCUSSION}

We have shown that the cryptococcal homologue of the CRZ1/SPl-like gene is required for slowdown of cryptococcal proliferation under reduced aeration, and its deletion increases susceptibility to cell wall stress and calcium stress. It remains unclear whether one or both of these defects cause the reduced fitness of the targeted deletion strain under hypoxic conditions. We believe in combined participation, because slowdown is beneficial
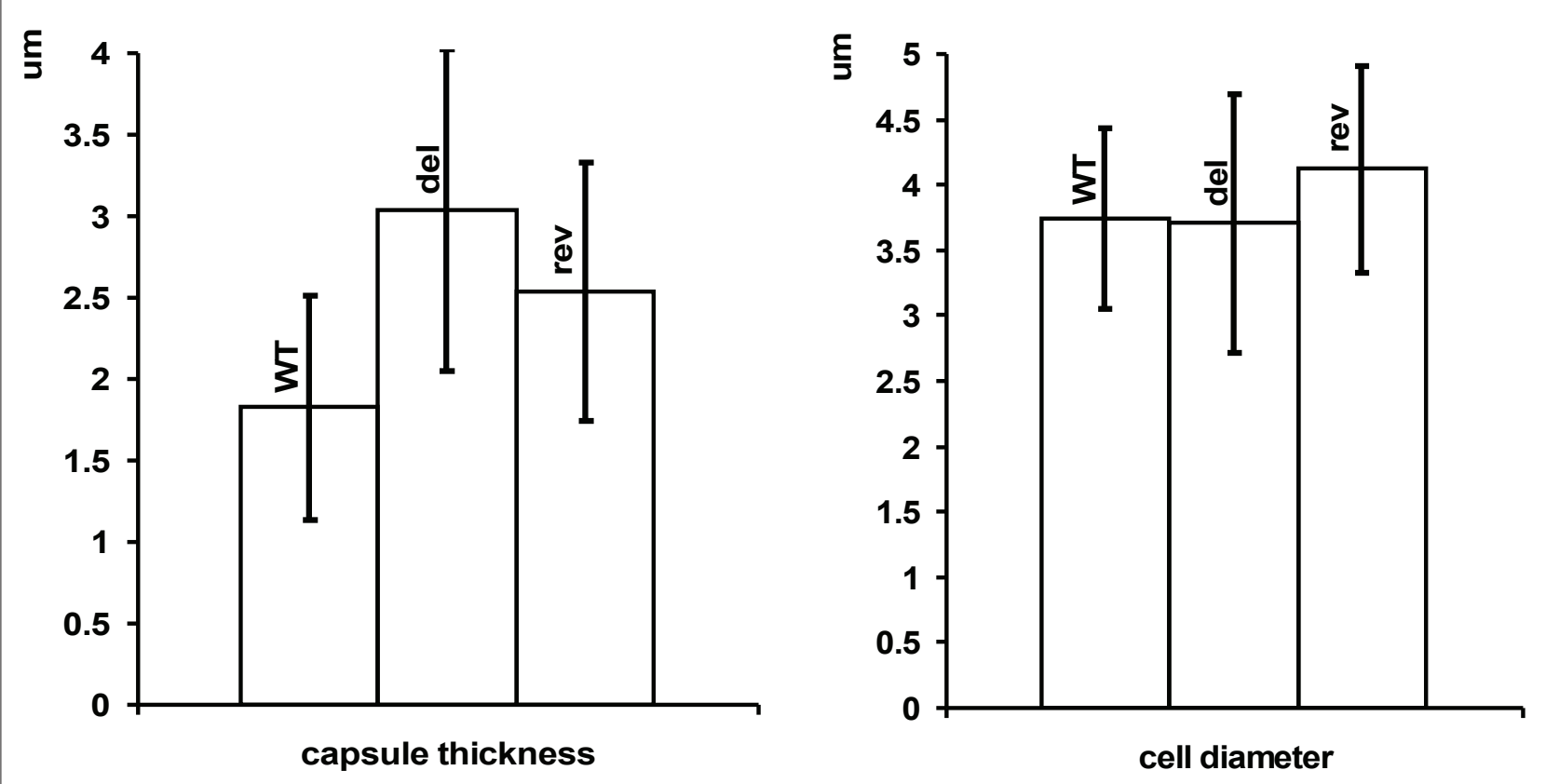

Fig. 6. Capsule induction assay on Rice extract agar.

Cells were grown on nutrient-poor Rice extract agar at $37^{\circ} \mathrm{C}$ under $5 \% \mathrm{CO}_{2}$ atmosphere for 3 days and capsules were visualized by India ink staining. Capsule thickness and cell diameter were measured on representative photographs. WT - wild-type, del = CNAG_00156.2 (CRZ1/SP1-like) gene targeted deletion strain, rev = reconstituted strain. 
to reduce oxygen consumption and thus to keep it in balance with reduced oxygen availability, while maintenance of cell integrity is energy demanding. Therefore, any cell integrity challenge, either by intrinsically impaired cell wall synthesis or by extrinsic damaging agents, should increase oxygen demand. Although unchallenged cells of the targeted deletion strain did not show any apparent phenotype, their colonies appeared glossy-shiny (data not shown), as already described ${ }^{21}$, indicating somehow impaired cell wall or capsule formation. In addition, the increased induction of capsule formation observed in the targeted deletion strain also indicates possible activation of compensatory mechanisms because of some latent cell wall damage caused by the $C R Z 1 / S P 1$-like gene deletion. Furthermore, the reduced ability of biofilm formation observed by us can be related to impaired cell wall construction as well, although the decreased ability to adapt to hypoxic conditions inside of biofilm body can also play its role. The latter link is further supported by the substantial overlap between the hypoxic and biofilm transcriptoms found in C. parapsilosis and C. albicans ${ }^{24,25}$ and other findings that demonstrate adaptation to hypoxia to be an integral component of biofilm formation in $C$. albicans ${ }^{26}$. We also have already suggested a possible link between biofilm-like adaptation to hypoxic environment in human tissue and the dormant state of cryptococcal infection earlier ${ }^{10}$.

Surprisingly, we observed increased resistance to fluconazole as a result of targeted deletion of the CRZ1/ $S P 1$-like gene. We can only speculate about the underlying mechanisms. In a recent study, $C$. albicans cells with increased chitin level that was induced by activation of the $\mathrm{Ca}^{2+} /$ calcineurin and PKCl-pathways were demonstrated to be less susceptible to caspofungin ${ }^{27}$. Therefore, it is rather conceivable that a latent cell wall damage resulting from the $C R Z 1 / S P 1$-like gene deletion activates compensatory response that results in increased resistance to fluconazole.

\section{CONCLUSIONS}

Taken together, our data clearly indicate that the cryptococcal homologue of the CRZ1/SPl-like gene plays important role in survival under limited aeration, resistance to cell wall damaging agents, susceptibility to fluconazole, and biofilm formation, thus linking hypoxic adaptation, cell integrity and biofilm production in the pathogenic yeast $C$. neoformans. However, the exact positioning of the protein in cryptococcal signalling cascades is still puzzling. It also remains to be clarified what its primary role in cellular processes is, i.e. whether proper $C R Z 1 / S P 1$ regulated cell wall synthesis is the key to hypoxic adaptation, or rather an adequate $C R Z 1 / S P 1$-mediated slowdown of proliferation is a prerequisite to successful survival under hypoxia, including proper cell wall construction. In our opinion, slowed proliferation should enable the cells to keep their oxygen consumption in balance with its availability and thus also helps them to keep their integrity under hypoxic conditions.

\section{ACKNOWLEDGEMENTS}

Czech Science Foundation (310/06/0645), Ministry of Education, Youth and Sports, Czech Republic (MSM6198959223) and the Internal Grant Agency of Palacky University Olomouc (LF_2013_012) supported this work. Infrastructural part of this project (Institute of Molecular and Translational Medicine) was supported from the Operational Programme Research and Development for Innovations (project CZ.1.05/2.1.00/01.0030). This work was also partially supported by Grants-in-Aid for Scientific Research from the Ministry of Education, Science, Sports, and Culture of Japan (to Susumu Kawamoto) and Cooperative Research Program of Medical Mycology Research Center, Chiba University (to Susumu Kawamoto). The support of the Japan Society for the Promotion of Science (JSPS) to Zuzana Moranova is highly acknowledged (JSPS Postdoctoral Fellowship for Foreign Researchers [Shortterm]). We thank Dr. J. Kwon-Chung for providing the $A$. tumefaciens strain EHA 105 harbouring kanamycin resistance plasmid pYCC716, and Dr. J. Heitman for providing the pJAF1 plasmid harbouring the neomycin resistance marker cassette.

\section{CONFLICT OF INTEREST STATEMENT}

The authors state that there are no conflicts of interest regarding the publication of this article.

\section{REFERENCES}

1. Idnurm A, Bahn YS, Nielsen K, Lin X, Fraser JA, Heitman J. Deciphering the model pathogenic fungus Cryptococcus neoformans. Nat Rev Microbiol 2005;3:753-64.

2. Odds FC, De Backer T, Dams G, Vranckx L, Woestenborghs F. Oxygen as limiting nutrient for growth of Cryptococcus neoformans. J Clin Microbiol 1995;33:995-7.

3. Takeo K, Tanaka R, Miyaji M, Nishimura K. Unbudded G2 as well as $\mathrm{G} 1$ arrest in the stationary phase of the basidiomycetous yeast Cryptococcus neoformans. FEMS Microbiol Lett 1995;129:231-5.

4. Ohkusu M, Hata K, Takeo K. Bud emergence is gradually delayed from $\mathrm{S}$ to $\mathrm{G} 2$ with progression of growth phase in Cryptococcus neoformans. FEMS Microbiol Lett 2001;194:251-5.

5. Ohkusu M, Raclavsky V, Takeo K. Deficit in oxygen causes G(2) budding and unbudded $\mathrm{G}(2)$ arrest in Cryptococcus neoformans. FEMS Microbiol Lett 2001;204:29-32.

6. Chun CD, Liu OW, Madhani HD. A link between virulence and homeostatic responses to hypoxia during infection by the human fungal pathogen Cryptococcus neoformans. PLoS Pathog 2007;3:e22.

7. Chang YC, Bien CM, Lee H, Espenshade PJ, Kwon-Chung KJ. Sre1p, a regulator of oxygen sensing and sterol homeostasis, is required for virulence in Cryptococcus neoformans. Mol Microbiol 2007;64:614-29.

8. Chang YC, Ingavale SS, Bien C, Espenshade P, Kwon-Chung KJ. Conservation of the sterol regulatory element-binding protein pathway and its pathobiological importance in Cryptococcus neoformans. Eukaryot Cell 2009;8:1770-9.

9. Ernst JF, Tielker D. Responses to hypoxia in fungal pathogens. Cell Microbiol 2009;11:183-90.

10. Moranova Z, Kawamoto S, Raclavsky V. Hypoxia sensing in Cryptococcus neoformans: Biofilm-like adaptation for dormancy? Biomed Pap Med Fac Univ Palacky Olomouc Czech Repub 2009;153:189-93. 
11. Grahl N, Cramer RA, Jr. Regulation of hypoxia adaptation: an overlooked virulence attribute of pathogenic fungi? Med Mycol 2010;48:1-15.

12. Grahl N, Shepardson KM, Chung D, Cramer RA. Hypoxia and Fungal Pathogenesis: To Air or Not to Air? Eukaryot Cell 2012;doi:10.1128/ EC.00031-12.

13. McClelland CM, Chang YC, Kwon-Chung KJ. High frequency transformation of Cryptococcus neoformans and Cryptococcus gattii by Agrobacterium tumefaciens. Fungal Genet Biol 2005;42:904-13.

14. Raclavsky V, Husickova V, Moranova Z, Ohkusu M, Fischer O, Precek J, Trtkova J, Takeo K, Kawamoto S. Growth strategy of the pathogenic yeast Cryptococcus neoformans submerged culture under different cultivation formats. Folia Microbiol (Praha) 2009;54:349-52.

15. Liu YG, Huang N. Efficient amplification of insert end sequences from bacterial artificial chromosome clones by thermal asymmetric interlaced PCR. Plant Molecular Biology Reporter 1998;16:175-81.

16. Davidson RC, Blankenship JR, Kraus PR, de Jesus Berrios M, Hul CM, D'Souza C, Wang P, Heitman J. A PCR-based strategy to generate integrative targeting alleles with large regions of homology. Microbiology 2002;148:2607-15.

17. Davidson RC, Cruz MC, Sia RA, Allen B, Alspaugh JA, Heitman J. Gene disruption by biolistic transformation in serotype $D$ strains of Cryptococcus neoformans. Fungal Genet Biol 2000;29:38-48.

18. Singer VL, Jones LJ, Yue ST, Haugland RP. Characterization of PicoGreen reagent and development of a fluorescence-based solution assay for double-stranded DNA quantitation. Anal Biochem 1997;249:228-38.

19. Costantino PJ, Budd DE, Gare NF. Enumeration of viable Candida albicans blastospores using tetrabromofluorescein (eosin Y) and flow cytometry. Cytometry 1995;19:370-5.
20. Walton FJ, Heitman J, Idnurm A. Conserved elements of the RAM signaling pathway establish cell polarity in the basidiomycete Cryptococcus neoformans in a divergent fashion from other fungi. Mol Biol Cell 2006;17:3768-80.

21. Adler A, Park YD, Larsen P, Nagarajan V, Wollenberg K, Qiu J, Myers TG, Williamson PR. A Novel Specificity Protein 1 (SP1)-like Gene Regulating Protein Kinase C-1 (Pkc1)-dependent Cell Wall Integrity and Virulence Factors in Cryptococcus neoformans. J Biol Chem 2011;286:20977-90.

22. Lev S, Desmarini D, Chayakulkeeree M, Sorrell TC, Djordjevic JT. The Crz1/Sp1 transcription factor of Cryptococcus neoformans is activated by calcineurin and regulates cell wall integrity. PLoS One 2012;7:e51403.

23. Raclavsky V, Novotny R, Smigova J, Vojkuvka Z. Nikkomycin Z counteracts Rylux BSU and Congo red inhibition of Saccharomyces cerevisiae growth but does not prevent formation of aberrant cell walls. Folia Microbiol (Praha) 1999;44:663-8.

24. Rossignol T, Ding C, Guida A, d'Enfert C, Higgins DG, Butler G. Correlation between biofilm formation and the hypoxic response in Candida parapsilosis. Eukaryot Cell 2009;8:550-9.

25. Stichternoth C, Ernst JF. Hypoxic adaptation by Efg1 regulates biofilm formation by Candida albicans. Appl Environ Microbiol 2009;75:3663-72.

26. Bonhomme J, Chauvel M, Goyard S, Roux P, Rossignol T, d'Enfert C. Contribution of the glycolytic flux and hypoxia adaptation to efficient biofilm formation by Candida albicans. Mol Microbiol 2011;80:995-1013.

27. Lee KK, Maccallum DM, Jacobsen MD, Walker LA, Odds FC, Gow NA, Munro CA. Elevated cell wall chitin in Candida albicans confers echinocandin resistance in vivo. Antimicrob Agents Chemother 2012;56:208-17. 